Research Article

\title{
Tripartite Evolutionary Game Model for Public Health Emergencies
}

\author{
Zhiqi Xu $\mathbb{D}^{1},{ }^{1}$ Yukun Cheng $\mathbb{D},{ }^{1}$ and Shuangliang Yao $\mathbb{D}^{2}$ \\ ${ }^{1}$ School of Business, Suzhou University of Science and Technology, Suzhou 215009, China \\ ${ }^{2}$ Jiangsu University of Science and Technology, Zhenjiang 212008, China \\ Correspondence should be addressed to Yukun Cheng; ykcheng@amss.ac.cn
}

Received 3 January 2021; Revised 8 April 2021; Accepted 15 April 2021; Published 18 May 2021

Academic Editor: Sundarapandian Vaidyanathan

Copyright $\odot 2021$ Zhiqi Xu et al. This is an open access article distributed under the Creative Commons Attribution License, which permits unrestricted use, distribution, and reproduction in any medium, provided the original work is properly cited.

Public health emergencies are more related to the safety and health of the public. For the management of the public health emergencies, all parties' cooperation is the key to preventing and controlling the emergencies. Based on the assumption of bounded rationality, we formulate a tripartite evolutionary game model, involving the local government, the enterprises, and the public, for the public health emergency, e.g., COVID-19. The evolutionary stable strategies under different conditions of the tripartite evolutionary game are explored, and the effect from different factors on the decision-makings of participants for public health emergencies is also analyzed. Numerical analysis results show that formulating reasonable subsidy measures, encouraging the participation of the public, and enforcing the punishment to enterprises for their negative behaviors can prompt three parties to cooperate in fighting against the epidemic. Our work enriches an understanding of the governance for the public health emergency and provides theoretical support for the local government and related participants to make proper decisions in public health emergencies.

\section{Introduction}

The COVID-19 [1] broke out at the end of 2019, which is highly infectious and has attracted significant attention from countries worldwide. On January 30, 2020, the World Health Organization (WHO) declared the novel coronavirus outbreak a Public Health Emergency of International Concern (PHEIC) [2]. It is difficult to curb the viral from spreading without vaccines or medicines [3], and many countries faced the dilemma of getting people and enterprises back to work orderly or not. The emergency governance of public health emergencies often involves many parts, such as the government, enterprises, and the public. Unreasonable regulations or untimely treatment may lead to the rapid spread of the epidemic and cause severe economic losses. How to effectively organize the members of society to participate the emergency governance in public health emergencies and to control the epidemic outbreak in a short time is of great significance to safeguard human life and health.

In the management of public health emergencies, there are three players in the game, including the local government, enterprises, and the public. They must find an optimal equilibrium mechanism to form a virtuous circle system in the game. In this paper, we construct a tripartite evolutionary game model, including the local government, enterprises, and the public, to study the management of public health emergencies. By leveraging the standard evolutionary game theory and necessary assumptions, the payoff matrix and corresponding replicator dynamic system are built, based on which the evolutionary stability strategies and their corresponding conditions are explored. Finally, we use numerical simulation to verify the evolution paths under the ideal states and discuss the impact of different parameters on the evolutionary processes of three parties' behaviors. 
For the public health emergencies, many research studies focus on the transmission of infectious diseases, change in the number of infected people, and the inflection points. They are mainly based on the mathematical models of epidemic diseases, including SIR models [4], SIS models [5, 6], SEIR models [7, 8], and so on. Many scholars describe the transmission of COVID-19 based on the SEIR model to analyze the effectiveness of prevention and put forward some corresponding countermeasures and suggestions. Hou et al. [9] applied the SEIR model to explore the effectiveness of the quarantine of Wuhan city against this epidemic, and then the results suggest that reducing the contact rate of latent individuals after quarantine and isolation can effectively reduce the number of individuals infected with COVID-19 and delay the peak time. An age-structured SEIR model is proposed by Prem et al. [10]; they found that physical distancing measures are effective, which can delay the peak and reduce the final size of the epidemic. Radulescu et al. [11] adopted the traditional SEIR model, and according to the simulation, they suggested that social distancing represents the most efficient strategy in curbing the effects of the outbreak. $\mathrm{Zu}$ et al. [12] constructed a compartmental model and estimated the efficacy of intervention strategies by sensitivity analysis and found that the quarantine measures the Chinese government adopted, such as postponing the relaxation of isolation, early diagnosis, broad close-contact tracing, and strict monitoring of infected persons, contribute to the control of the COVID-19.

In the field of management, game theory provides an efficient method for emergency decision-making to explore the social interaction among different participants [13]. Wang et al. [14] applied a noncooperative complete information game model for resource allocation during the multiple emergencies. Yang and $\mathrm{Xu}$ [15] analyzed the relationship between the decision-makers and an emergency, and then formulated an engineering model based on a sequential game. To address traffic network congestion to speed up the disaster response activity during a disaster, Purohit et al. [16] proposed a nondeterministic, noncooperative $n$-person game. Li [17] used evolutionary game theory and established an emergency management cooperation model to improve the efficiency of emergency management of the Middle Route of the South-to-North Water Diversion Project.

The existing research studies focus on analyzing the spread of the epidemic and the effectiveness of measures from the mathematical models of epidemic diseases; however, the studies on the dynamic decision-making of different participants in the epidemic particularly the cooperation among the local governments, enterprises, and the public by the evolutionary game model are not too much. The regulations to prevent the epidemic formulated by the local government can affect the strategy selections of enterprises and the public, which in addition affect the transmission of the epidemic. Due to the sudden outbreak of the epidemic, time left for participants to make judgments is relatively short, and the selected strategies can only be adjusted continuously according to the epidemic situation and the awareness of the epidemic of different parts. The strategy selection process conforms to the assumption of "bounded rationality," in which participants adopt imitative behaviors in the evolutionary game. Thus, we construct a tripartite evolutionary game model including the local government, enterprises, and the public, to analyze the dynamic adjustment process of the behaviors of these three parts and explore how the parameters impact their strategy selection so as to provide decision-making support for the local government and other participants.

The rest part of this paper is organized as follows. In Section 2, a three-part evolutionary game model is constructed and some necessary assumptions are introduced. We apply the standard method to analyze the behaviors among three parts to obtain the evolutionary stable strategies, and the corresponding conditions are explored in Section 3. Section 4 provides numerical experiments to illustrate the impact of parameters on the behavior selection in the evolutionary game model, and the last section draws conclusions and puts forward corresponding suggestions.

\section{The Evolutionary Game Consisting of Tripartite Participants}

2.1. Tripartite Evolutionary Game Model. In this paper, we mainly study the strategic interactions among the local government, enterprises, and the public during a public health emergency, by modeling a tripartite evolutionary game.

When a public health emergency occurs, the local government, as the formulator of the regulations to prevent and control the epidemic, has two kinds of strategies: one is strict governance $(\mathrm{G})$ and the other is nonstrict governance (NG). If the local government adopts the strategy of strict governance, then it would take active actions based on adequate knowledge of the epidemic, formulate the regulations on epidemic prevention, and then strictly supervise the implementation of these regulations. For example, Wuhan local government took the strictest measures to seal its borders from the rest of China on Jan 23, 2020 [18], which effectively constrained the spread of COVID-19. The strategy of nonstrict governance means that the local government does not pay enough attention to the epidemic and fails to formulate effective prevention and control measures.

During the epidemic, enterprises have two kinds of strategies: one is to strictly implement (I) the regulation, which are formulated by the local government and the other is nonstrict implementation (NI). When an enterprise follows the regulation, it would implement the prevention and control measures strictly, which brings it a certain cost and revenue losses. On the contrary, if it does not implement the regulations strictly, then it may get more revenues at the expense of the health of the public and the security of the society.

The public, an essential participant in the evolutionary game, plays an important role in the supervision of enterprises' behaviors. Thus, the public has two kinds of strategies: one is supervision (S) and the other is nonsupervision (NS). The strategy of supervision means that the public would supervise the enterprises strictly and disclose irregularities of 
enterprises in time if they exist, from the perspective of society security. The public may choose not to supervise because of the high cost or other reasons.

2.2. Basic Assumptions. In order to analyze the decisionmaking of the three parties, some basic assumptions are set in advance as follows.

Assumption 1. . The local government will incur the cost, denoted by $C_{1}$, to offer strict governance since it shall conduct exhaustive investigations to make a further recognition of the virus and epidemic. Correspondingly, the cost of nonstrict governance is denoted by $C_{2}$ with $C_{2}<C_{1}$. If the local government takes rapid actions on the epidemic and formulates the effective regulations, then the public will praise the government's behavior, which can enhance the credibility of the government and such a positive effect is denoted by $W^{G}$. To encourage the behavior of strict implementation, the government will provide subsidies to enterprises due to their positive behaviors. On the contrary, if enterprises do not implement the prevention and control measures strictly, then they would bring high risk of epidemic further spreading, in addition harm the health of public. The government must bear the losses from the deteriorated epidemic situation and the external public pressure, which is denoted by $L^{G}$. Then, the government imposes a penalty of $P$ on enterprises, once their behaviors of nonstrict implementation are disclosed by the public or found out by the government. Particularly, we assume that the nonstrict implementation behaviors must be found out by the government if the government selects the strict governance strategy. Otherwise, such negative behaviors will be disclosed by the public if the public supervises the enterprises.

Assumption 2. . If the enterprises strictly implement the prevention and control measures, such as purchasing enough protection articles, suspending production, shortening working hours during the epidemic, and so on, then it will carry a cost of $C_{3}$ to the enterprises, and the corresponding revenue is denoted by $R_{1}$. At the same time, these enterprises' positive behaviors help them win a high reputation, which is quantified as $W^{E}$, and obtain the subsidy of $\alpha C_{3}$ provided by the local government. Here, the parameter $\alpha(0<\alpha<1)$ reflects the strength of encouragement from the government. Under the circumstances that the government does not govern strictly and the public does not supervise, they will not care about the enterprises' strategy even if they strictly carry out epidemic prevention, and the enterprises' reputation will not be improved at this time. If the enterprises decide not to implement strictly, then they would bear a less cost $C_{4}\left(<C_{3}\right)$ and a higher revenue $R_{2}\left(>R_{1}\right)$. However, these negative behaviors also make the enterprises suffer a penalty $P$ from the local government.

Assumption 3. . Considering the safety and the health, the public may take the initiative to supervise the enterprises based on their scientific cognition of the epidemic situation.
The cost from the supervision is denoted by $C_{5}(>0)$, and we assume that the cost is zero if the public does not supervise. When the local government chooses the "strict governance" strategy, the behavior of supervision is encouraged, and thus the public will receive the award of $H$ from the local government. Under the strategy profile (NG, NI, S), in which the local government selects the strategy of nonstrict governance, the enterprises do not implement the regulations while the public supervises the enterprises, then the enterprises' "nonimplementation" behaviors will be disclosed by the public, and then the government shall share a part of penalty denoted by $\beta P(0<\beta<1)$ with the public to encourage its supervision behavior. Of course, the behaviors of nonstrict implementation by the enterprises will harm the health of the public and thus bring the losses $L^{P}$ to the public.

The model parameters are summarized in Table 1.

2.3. The Payoff Matrix. In summary, the local government, enterprises, and the public belong to our tripartite evolutionary game with finite rationality. The previously discussed assumptions reveal that under the strict governance, the government will pay the costs for its governance actions, the subsidies to enterprises, and the rewards to the public, and enterprises will be punished due to their nonstrict implementation behaviors. If the strategy of strict implementation is adopted by enterprises, then they will earn the government's encouragement and high reputations. However, if enterprises do not strictly implement the regulations, then such negative behaviors will damage the safety of society, leading to the losses to the government and the public, and the government will punish enterprises. In addition to paying the supervising cost, the public will earn the rewards from the government or share a part of penalty from enterprises.

Given the above discussions and assumptions, the payoffs of tripartite participants can be derived under different strategy profiles, and thus the payoff matrix of the tripartite evolutionary game is displayed in Table 2.

Without loss of generality, we assume

$$
\left(R_{1}+\alpha C_{3}+W^{E}-C_{3}\right)-\left(R_{2}-P-C_{4}\right)>0,
$$

which means that the enterprises' payoff of strict implementation is higher than the one of nonstrict implementation, under the condition that the local government takes the strategy of strict governance.

\section{Equilibrium Analysis}

Suppose that the probability of the local government choosing "strict governance" strategy in public health emergencies is $x(0 \leq x \leq 1)$, and then the probability of choosing "nonstrict governance" strategy is $1-x$. Variable $y(0 \leq y \leq 1)$ represents the probability of enterprises choosing the "strict implementation" strategy, and then the probability of choosing the "nonstrict implementation" strategy is $1-y$. The probability of the public to supervise is denoted by $z(0 \leq z \leq 1)$, and then $1-z$ is the probability not to supervise. 
TABLE 1: Parameters in the tripartite evolutionary game for public health emergencies.

\begin{tabular}{lc}
\hline Parameters & Description \\
\hline$C_{1}$ & Strict governance cost of the local government \\
$C_{2}$ & Nonstrict governance cost of the local government $\left(C_{1}>C_{2}\right)$ \\
$C_{3}$ & Strict implementation cost of the enterprises \\
$C_{4}$ & Nonstrict implementation cost of the enterprises $\left(C_{3}>C_{4}\right)$ \\
$\alpha C_{3}$ & Enterprises' subsidy from the local government $(0 \leq \alpha \leq 1)$ because of strict implementation \\
$C_{5}$ & Supervision cost of the public \\
$P$ & Enterprises' penalty from the local government because of nonstrict implementation \\
$L^{G}$ & Losses to the local government because of enterprises' nonstrict implementation \\
$L^{P}$ & Losses to the public because of enterprises' nonstrict implementation \\
$R_{1}$ & Enterprises' revenue when adopting strict implementation strategy \\
$R_{2}$ & Enterprises' revenue when adopting nonstrict implementation strategy $\left(R_{1}<R_{2}\right)$ \\
$W^{G}$ & Reputation of the local government because of strict governance behaviors \\
$W^{E}$ & Reputation of enterprises because of strict implementation behaviors \\
$\beta$ & The coefficient of the penalty $(0<\beta<1)$ \\
$H$ & Rewards to the public from the local government due to the public's supervision behavior \\
\hline
\end{tabular}

TABLE 2: The payoff matrix of the model.

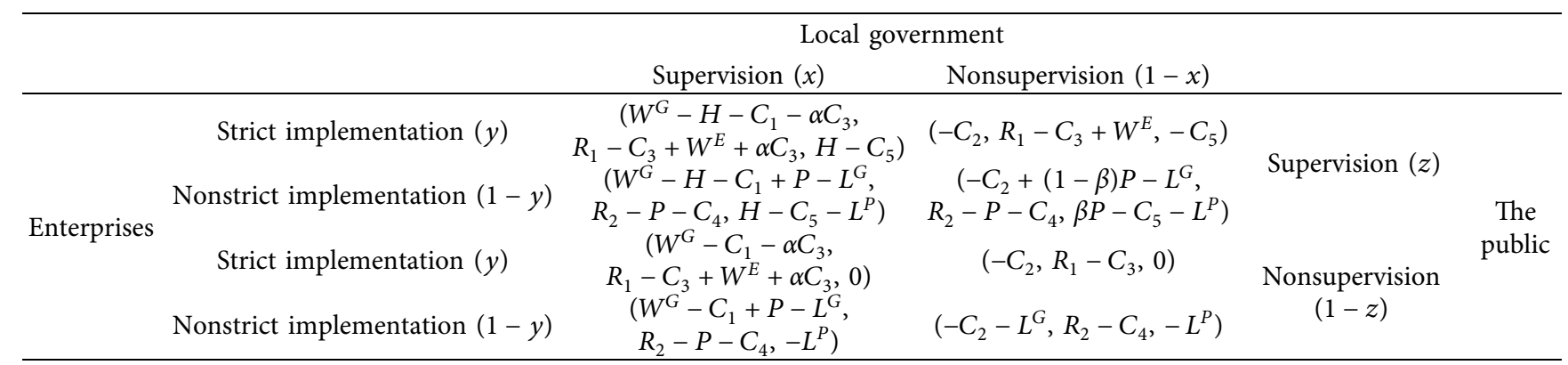

Note: From left to right are the respective benefits of the local government, enterprises, and the public.

\subsection{Stability Strategy Analysis of Three Parties}

3.1.1. The Analysis for the Local Government. The expected payoff $U_{G 1}$ of the local government when it chooses the "strict governance" strategy is

$$
\begin{aligned}
U_{G 1}= & y z\left(W^{G}-H-C_{1}-\alpha C_{3}\right)+y(1-z)\left(W^{G}-C_{1}-\alpha C_{3}\right) \\
& +(1-y) z\left(W^{G}-H-C_{1}+P-L^{G}\right)+(1-y)(1-z)\left(W^{G}-C_{1}+P-L^{G}\right)
\end{aligned}
$$

and the expected payoff of the local government when it chooses the behavior of "nonstrict governance" is

$$
\begin{aligned}
U_{G 2}= & y z\left(-C_{2}\right)+y(1-z)\left(-C_{2}\right)+(1-y) z\left(-C_{2}+(1-\beta) P-E_{1}\right) \\
& +(1-y)(1-z)\left(-C_{2}-E_{1}\right) .
\end{aligned}
$$

Thus, the local government's average expected payoff is

$$
\overline{U_{G}}=x U_{G 1}+(1-x) U_{G 2},
$$

and the replicator dynamic equation of the local government is 


$$
\begin{aligned}
F(x) & =\frac{\mathrm{d} x}{\mathrm{~d} t}=x\left(U_{G 1}-\overline{U_{G}}\right)=x(1-x)\left(U_{G 1}-U_{G 2}\right) \\
& =x(1-x)\left\{[(1-\beta) P y-H-(1-\beta) P] z+\left(-\alpha C_{3}-P\right) y+W^{G}-C_{1}+P+C_{2}\right\} .
\end{aligned}
$$

Let us derive $F(x)$ relative to $x$, and the outcome is as follows:

$$
\frac{\mathrm{d} F(x)}{\mathrm{d} x}=(1-2 x)\left\{[(1-\beta) P y-H-(1-\beta) P] z+\left(-\alpha C_{3}-P\right) y+W-C_{1}+P+C_{2}\right\}
$$

Let $\quad z_{0}=\left(-\left(\alpha C_{3}+P\right) y+W^{G}-C_{1}-P+C_{2} /(1-\beta)\right.$ $P(1-y)+H)$. So,

(1) If $z=z_{0}$, then $F(x) \equiv 0$. No matter what $x$ is, it is always in a stable state.

(2) If $z \neq z_{0}$, then let $F(x)=0$, and then two stable points $x=0$ and $x=1$ are obtained.

According to the stability theorem and the dynamic game theory of replication $[19,20], x^{*}$ is an evolutionary stable strategy (ESS) point if the conditions of $F\left(x^{*}\right)=0$ and $\left(\mathrm{d} F\left(x^{*}\right) / \mathrm{d} x\right)<0$ are satisfied. Based on this claim, we can get the following.

Claim 1. If $0<z<z_{0}$, then "strict governance" strategy is the ESS of the local government. If $z_{0}<z<1$, then "nonstrict governance" strategy is the ESS of the local government.

Proof 1. It is not hard to see that when $0<z<z_{0}$, we have $\left.(\mathrm{d} F(x) / \mathrm{d} x)\right|_{x=0}>0$ and $\left.(\mathrm{d} F(x) / \mathrm{d} x)\right|_{x=1}<0$, indicating $x=1$ is the ESS. When $z_{0}<z<1$, then $\left.(\mathrm{d} F(x) / \mathrm{d} x)\right|_{x=0}<0$ and $\left.(\mathrm{d} F(x) / \mathrm{d} x)\right|_{x=1}>0$, showing $x=0$ is the ESS.

Proposition 1. If the local government has a higher reputation, then it tends to adopt "strict governance" strategy. If the cost of "strict governance" strategy $C_{1}$ increases or the cost of "nonstrict governance" strategy $C_{2}$ decreases, then the strategy of the local government may incline to be "nonstrict governance" due to the cost pressure.
Proof 2. The dynamic evolution for the local government is demonstrated in Figure 1. The whole space is divided into two parts by the inner surface $z=z_{0}$. When the initial state $(x, y, z)$ is in region II, showing $0<z<z_{0}$, then $x=1$ is the ESS, indicating the strategy of "strict governance" is the eventual choice of the local government. When the initial state $(x, y, z)$ is in region $\mathrm{I}$, corresponding to the case of $z_{0}<z<1$, then $x=0$ is the ESS, implying the local government will choose the "nonstrict governance" strategy eventually. The volumes of region I and II represent the probabilities of the local government choosing the "nonstrict governance" strategy and "strict governance" strategy, respectively. It reveals that the value of $z_{0}$ increases with the reputation $W^{G}$ of the local government, and thus the volume of region II increases with $W^{G}$ if other variables are fixed. It means that the local government tends to adopt "strict governance" strategy with a higher reputation. Furthermore, when the cost of "strict governance" strategy $C_{1}$ increases or the cost of "nonstrict governance" strategy $C_{2}$ decreases, the volume of region II will decrease since $z_{0}$ is smaller, and thus the strategy of the local government is inclined to be "nonstrict governance" due to the cost pressure.

3.1.2. The Analysis for Enterprises. We use $U_{E 1}$ and $U_{E 2}$ to denote the expected payoffs of the enterprises when the enterprises choose the strategies of "strict implementation" and "nonstrict implementation," respectively. Their detailed formulas are

$$
\begin{aligned}
U_{E 1}= & x z\left(R_{1}-C_{3}+W^{E}+\alpha C_{3}\right)+x(1-z)\left(R_{1}-C_{3}+W^{E}+\alpha C_{3}\right) \\
& +(1-x) z\left(R_{1}-C_{3}+W^{E}\right)+(1-x)(1-z)\left(R_{1}-C_{3}\right) \\
U_{E 2}= & x z\left(R_{2}-P-C_{4}\right)+x(1-z)\left(R_{2}-P-C_{4}\right)+(1-x) z\left(R_{2}-P-C_{4}\right)+(1-x)(1-z)\left(R_{2}-C_{4}\right)
\end{aligned}
$$

Therefore, the enterprises' average expected payoff is calculated as

$$
\overline{U_{E}}=y U_{E 1}+(1-y) U_{E 2} \text {, }
$$

and the corresponding replicator dynamic equation is 


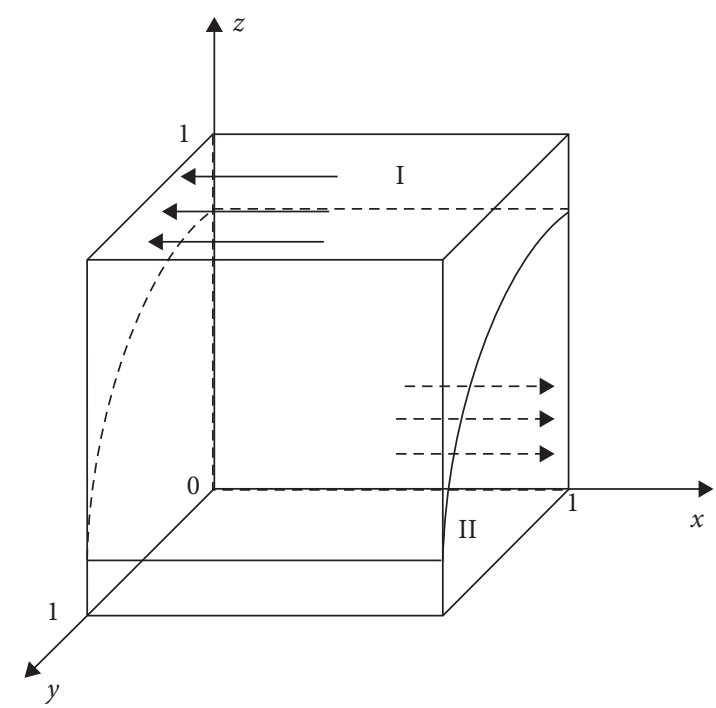

Figure 1: The local government's dynamic evolution.

$$
\begin{aligned}
G(y)= & \frac{\mathrm{d} y}{\mathrm{~d} t}=y\left(U_{E 1}-\overline{U_{E}}\right)=y(1-y)\left(U_{E 1}-U_{E 2}\right)=y(1-y)\left\{\left[-z\left(P+W^{E}\right)+\alpha C_{3}+P+W^{E}\right] x\right. \\
& \left.+z\left(P+W^{E}\right)+R_{1}-C_{3}-R_{2}+C_{4}\right\} .
\end{aligned}
$$

Let us continue to compute the derivative of $G(y)$, then

$$
\frac{\partial G(y)}{\partial y}=(1-2 y)\left\{\left[-z\left(P+W^{E}\right)+\alpha C_{3}+P+W^{E}\right] x+z\left(P+W^{E}\right)+R_{1}-C_{3}-R_{2}+C_{4}\right\}
$$

Let $\quad x_{0}=\left(-z\left(P+W^{E}\right)-R_{1}+C_{3}+R_{2}-C_{4} /(1-z)\right.$ $\left.\left(P+W^{E}\right)+\alpha C_{3}\right)$. So,

(1) If $x=x_{0}$, then $G(y) \equiv 0$, meaning that any $y \in[0,1]$ is the stable point

(2) If $x \neq x_{0}$, then there are only two stable points $y=0$ and $y=1$, ensuring $G(y)=0$

Claim 2. If $0<x<x_{0}$, then "nonstrict implementation" strategy is the ESS of the enterprises. If $x_{0}<x<1$, then "strict implementation" strategy is the ESS of the enterprises.

Proof 3. It is not hard to see that when $0<x<x_{0}$, we have $\left.(\mathrm{d} G(y) / \mathrm{d} y)\right|_{y=0}<0$ and $\left.(\mathrm{d} G(y) / \mathrm{d} y)\right|_{y=1}>0$, and thus $y=0$ is the ESS. When $x_{0}<x<1$, then $\left.(\mathrm{d} G(y) / \mathrm{d} y)\right|_{y=0}>0$ and $\left.(\mathrm{d} G(y) / \mathrm{d} y)\right|_{y=1}<0$ implying $y=1$ is the ESS.

Proposition 2. When the punishment $P$, the reputation $W^{E}$, or the subsidy factor $\alpha$ increases, the enterprises are more inclined to adopt the "strict implementation" strategy.
Proof 4. Figure 2 illustrates the dynamic evolutionary process of the enterprises. The whole space is partitioned into two parts by the inner surface $x=x_{0}$. When the initial state $(x, y, z)$ is in region III, showing $x>x_{0}$, then $y=1$ is the ESS, indicating that the enterprises eventually choose the "strict implementation" strategy. When the initial state $(x, y, z)$ is in region IV, which corresponds to the case of $x_{0}<x$, then $y=0$ is the ESS. It implies that the "nonstrict implementation" strategy is the enterprises' eventual choice. Further analysis indicates that when the punishment $P$, the reputation $W^{E}$, or the subsidy factor $\alpha$ increases, $x_{0}$ will decrease, leading to the increase in region III's volume and the decrease in region IV's volume. Thus, the enterprises are more inclined to adopt the "strict implementation" strategy.

3.1.3. The Analysis for the Public. Similarly, we denote the expected payoffs of the public when choosing "supervision" strategy or "nonsupervision" strategy by $U_{P 1}$ or $U_{P 2}$, respectively.

$$
\begin{aligned}
& U_{P 1}=x y\left(H-C_{5}\right)+x(1-y)\left(H-C_{5}-E_{2}\right)+(1-x) y\left(-C_{5}\right)+(1-x)(1-y)\left(\beta P-C_{5}-E_{2}\right), \\
& U_{P 2}=x(1-y)\left(-E_{2}\right)+(1-x)(1-y)\left(-E_{2}\right) .
\end{aligned}
$$




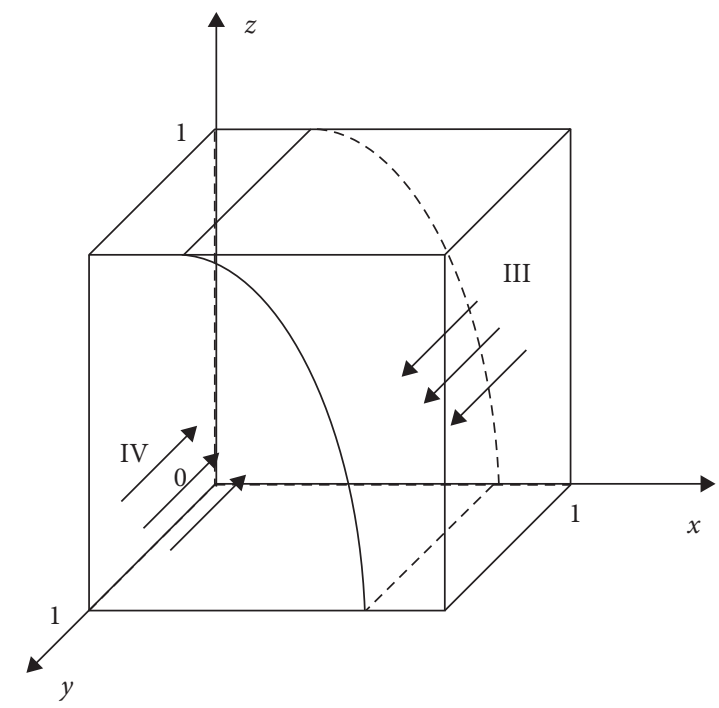

FIgURE 2: The enterprises' dynamic evolution.

Hence, the average expected payoff of the public is denoted by $\overline{U_{P}}$, where

$$
\overline{U_{P}}=z U_{31}+(1-z) U_{32} .
$$

$$
Q(z)=\frac{\mathrm{d} z}{\mathrm{~d} t}=z(1-z)\left(U_{31}-U_{32}\right)=z(1-z)\left[-\beta P(1-x) y+(H-\beta P) x+\beta P-C_{5}\right]
$$

whose derivative is shown as follows:

$$
\frac{\partial Q(z)}{\partial z}=(1-2 z)\left[-\beta P(1-x) y+(H-\beta P) x+\beta P-C_{5}\right]
$$

Let $y_{0}=\left((H-\beta P) x+\beta P-C_{5} / \beta P(1-x)\right)$. Then,

(1) If $y=y_{0}$, then $Q(z) \equiv 0$ and any $z \in[0,1]$ is in a stable point

(2) If $y \neq y_{0}$, then there are two stable points $z=0$ and $z=1$, ensuring $Q(z)=0$

Claim 3. If $0<y<y_{0}$, then "supervision" is the ESS of the public. If $y_{0}<y<1$, then "nonsupervision" is the ESS of the public.

Proof 5. When $0<y<y_{0}$, we have $\left.(\mathrm{dQ}(z) / \mathrm{d} z)\right|_{z=0}>0$ and $\left.(\mathrm{dQ}(z) / \mathrm{d} z)\right|_{z=1}<0$, and thus $z=1$ is the ESS. When $y_{0}<y<1$, then $\left.(\mathrm{d} Q(z) / \mathrm{d} z)\right|_{z=0}<0$ and $\left.(\mathrm{dQ}(z) / \mathrm{d} z)\right|_{z=1}>0$, implying $z=0$ is the ESS.

Proposition 3. If the coefficient $\beta$ of penalty or the reward $H$ increases, the public will tend to supervise, and when the cost for the public to supervise increases, then the public eventually chooses the "nonsupervision" strategy.
Proof 6. The dynamic evolution process of the public's decision is displayed in Figure 3. In this figure, the whole space is divided into region $\mathrm{V}$ and region VI. If the initial state $(x, y, z)$ is in region $\mathrm{V}$, indicating $y<y_{0}$, then $z=1$ is the ESS and the public tend to adopt the "supervision" strategy. When the initial state $(x, y, z)$ is in region VI, which corresponds to the case of $y>y_{0}$, then $z=0$ is the ESS and the "nonsupervision" strategy will be the eventual choice of the public. Particularly, $y_{0}$ can be rewritten as $y_{0}=1+\left(H x-C_{5} / \beta P(1-x)\right) \leq 1$, and thus $H x-C_{5} \leq 0$ and $x \leq \min \left\{1,\left(C_{5} / H\right)\right\}$. It is not hard to see if the coefficient $\beta$ of penalty or the reward $H$ increases, $y_{0}$ increases too and thus the volume of region $\mathrm{V}$ becomes larger. Under this case, the public will tend to supervise. When the $\operatorname{cost} C_{5}$ for the public to supervise increases, then $y_{0}$ decreases, leading to the decrease in region VI's volume. Then, the cost pressure prompts the public to choose the "nonsupervision" strategy.

3.2. Solving Evolutionary Stability Strategy Based on the Replicator Dynamic System. In this section, we propose the analysis for the evolutionary stable strategies under the mutual interaction among the local government, the enterprises, and the public. For this purpose, we first integrate three replicator dynamic equations (5)-(13) together to build a tripartite replicator dynamic system for the public health emergencies. 


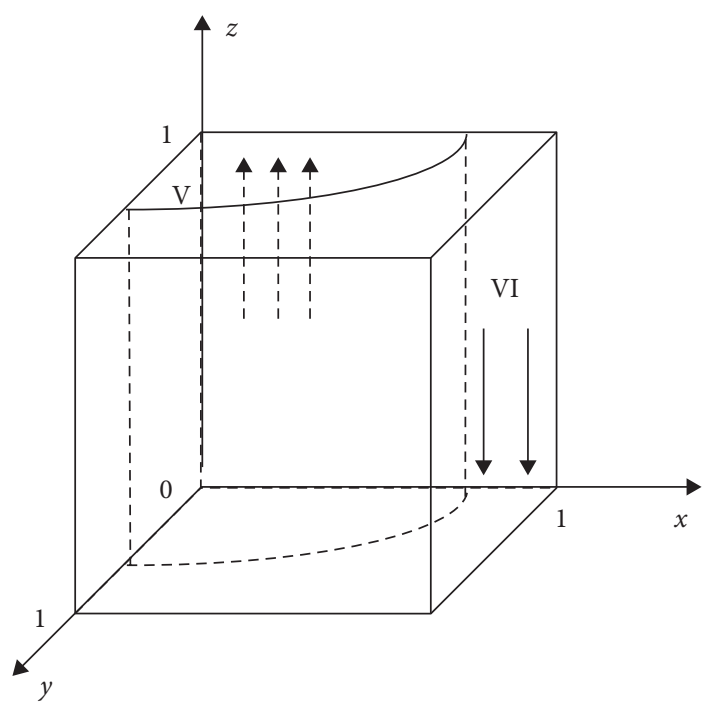

FIgURE 3: The public's dynamic evolution.

$$
\left\{\begin{array}{l}
F(x)=x(1-x)\left\{[(1-\beta) P y-H-(1-\beta) P] z+\left(-\alpha C_{3}-P\right) y+W^{G}-C_{1}+P+C_{2}\right\} \\
G(y)=y(1-y)\left\{\left[-z\left(P+W^{E}\right)+\alpha C_{3}+P+W^{E}\right] x+z\left(P+W^{E}\right)+R_{1}-C_{3}-R_{2}+C_{4}\right\} \\
Q(z)=z(1-z)\left[-\beta P(1-x) y+(H-\beta P) x+\beta P-C_{5}\right] .
\end{array}\right.
$$

Lemma 1. The replicator dynamic system (15) has eight equilibrium points, which are $E_{1}(0,0,0), E_{2}(0,0,1)$, $E_{3}(0,1,0), E_{4}(0,1,1), E_{5}(1,0,0), E_{6}(1,0,1), E_{7}(1,1,0)$, and $E_{8}(1,1,1)$.

Proof 7. Let $F(x)=0, G(y)=0$, and $Q(z)=0$; simultaneously, we can get nine equilibrium points of system (15).
They are $E_{1}(0,0,0), E_{2}(0,0,1), E_{3}(0,1,0), E_{4}(0,1,1)$, $E_{5}(1,0,0), \quad E_{6}(1,0,1), \quad E_{7}(1,1,0), \quad E_{8}(1,1,1)$, and $E_{9}\left(x^{*}, y^{*}, z^{*}\right)$, respectively, where point $E_{9}\left(x^{*}, y^{*}, z^{*}\right)$ is just the solution, satisfying the following equations simultaneously:

$$
\left\{\begin{array}{l}
{[(1-\beta) P y-H-(1-\beta) P] z+\left(-\alpha C_{3}-P\right) y+W^{G}-C_{1}+P+C_{2}=0,} \\
{\left[-z\left(P+W^{E}\right)+\alpha C_{3}+P+W^{E}\right] x+z\left(P+W^{E}\right)+R_{1}-C_{3}-R_{2}+C_{4}=0} \\
-\beta P(1-x) y+(H-\beta P) x+\beta P-C_{5}=0 .
\end{array}\right.
$$

Clearly, $E_{1}$ to $E_{8}$ are pure Nash equilibria, while point $E_{9}$ is a mixed Nash equilibrium. By the results of Ritzberger [21] and Selten [22], only the strict Nash equilibria are asymptotically stable in multipopulation evolutionary game. Because any strict Nash equilibrium must be a pure strategy, it is enough for us to discuss the stability of equilibrium solutions $E_{1}$ to $E_{8}$, and $E_{9}$ cannot be stable.

We leverage the Jacobian matrix of system (15), shown as follows, to analyze the stability of each Nash equilibrium solution:

$$
J=\left[\begin{array}{ccc}
\frac{\partial F(x)}{\partial x} & \frac{\partial F(x)}{\partial y} & \frac{\partial F(x)}{\partial z} \\
\frac{\partial G(y)}{\partial x} & \frac{\partial G(y)}{\partial y} & \frac{\partial G(y)}{\partial z} \\
\frac{\partial Q(z)}{\partial x} & \frac{\partial Q(z)}{\partial y} & \frac{\partial Q(z)}{\partial z}
\end{array}\right] .
$$

To be specific, each element in the Jacobian matrix $J$ is expressed as follows: 


$$
\begin{aligned}
& \frac{\partial F(x)}{\partial x}=(1-2 x)\left\{[(1-\beta) P y-H-(1-\beta) P] z+\left(-\alpha C_{3}-P\right) y+W^{G}-C_{1}+P+C_{2}\right\} ; \\
& \frac{\partial F(x)}{\partial y}=x(1-x)\left[(1-\beta) P y-\alpha C_{3}-P\right] ; \\
& \frac{\partial F(x)}{\partial z}=x(1-x)[(1-\beta) P y-H-(1-\beta) P] ; \\
& \frac{\partial G(y)}{\partial x}=y(1-y)\left[-z\left(P+W^{E}\right)+\alpha C_{3}+P+W^{E}\right] ; \\
& \frac{\partial G(y)}{\partial y}=(1-2 y)\left\{\left[-z\left(P+W^{E}\right)+\alpha C_{3}+P+W^{E}\right] x+z\left(P+W^{E}\right)+R_{1}-C_{3}-R_{2}+C_{4}\right\} ; \\
& \frac{\partial G(y)}{\partial z}=y(1-y)(1-x)\left(P+W^{E}\right) ; \\
& \frac{\partial Q(z)}{\partial x}=z(1-z)[-\beta P y+H-\beta P] ; \\
& \frac{\partial Q(z)}{\partial y}=-z(1-z)(1-x) \beta P ; \\
& \frac{\partial Q(z)}{\partial z}=(1-2 z)\left[-\beta P(1-x) y+(H-\beta P) x+\beta P-C_{5}\right] .
\end{aligned}
$$

Let us compute the eigenvalues of $J$ at each of eight equilibrium points, and the corresponding eigenvalues are all listed in Table 3.

Theorem 1. The four equilibrium points $(0,0,0),(0,0,1),(1,1,0)$, and $(1,1,1)$ are the ESS if the stability conditions in Table 4 are satisfied.

Proof 8. By Lyapunov system stability theory [23], a Nash equilibrium solution is an ESS, if and only if at this point, all eigenvalues of the Jacobian matrix are negative. Because $R_{2}>R_{1}$ and $C_{3}>C_{4}$, we have the second eigenvalue $\lambda_{2}$ at point $E_{3}(0,1,0)$ is positive. The third eigenvalue $\lambda_{3}$ at point $E_{4}(0,1,1)$ is positive too, as $C_{5}>0$. In addition, due to the Assumption 1, it is obvious that the second eigenvalue $\lambda_{2}$ at point $E_{5}(1,0,0)$ and $E_{6}(1,0,1)$ is both positive. Therefore, these four equilibrium points $(0,1,0),(0,1,1),(1,0,0)$, and $(1,0,1)$ cannot be the ESS of the system.

If the conditions in Case 1 are all satisfied, which are equivalent to $W^{G}+P<C_{1}-C_{2}, \quad R_{1}-C_{3}<R_{2}-C_{4}$, and $\beta P<C_{5}$, then equilibrium point $E_{1}(0,0,0)$ is the ESS. The strategies in $E_{1}(0,0,0)$ are "nonstrict governance" from the local governments, "nonimplementation" from the enterprises, and "nonsupervision" from the public. From the conditions in Case 1, we can observe that the low reputation $W^{G}$ and high strict governance cost $C_{1}$ prompt the local government to loosen its governance; since the net profit of $R_{1}-C_{3}$ when the enterprises strictly implement is lower than the one of $R_{2}-C_{4}$ when the "nonstrict implementation" strategy is adopted, the enterprises tend to behave negatively to pursue high profit; in addition, the public would not supervise because of the higher cost $C_{5}$ of supervision and the small rewards $\beta P$. In this case, the whole system is entirely laissez-faire, causing rapid outbreak of the epidemic and endangering the security of the public, society, and even the whole country.

If the conditions in Case 2 are all satisfied, which are equivalent to $W^{G}-C_{1}-H+P-L^{G}<(1-\beta) P-C_{2}-L^{G}$, $R_{1}-C_{3}+W^{E}<R_{2}-C_{4}-P$, and $C_{5}<\beta P$, then $E_{2}(0,0,1)$ is the ESS. The strategies in $E_{2}(0,0,1)$ are that the local government does not strictly govern, the enterprises do not strictly implement, and the public adopts "supervision" strategy. From the conditions in Case 2, we can observe that the payoff $\left(=(1-\beta) P-C_{2}-L^{G}\right)$ of the local government from nonstrict governance is higher than that $\left(=W^{G}-C_{1}-\right.$ $\left.H+P-L^{G}\right)$ from strict governance; the enterprises would get higher payoff when they choose the "nonstrict implementation" strategy due to the condition $R_{1}-C_{3}+W^{E}<R_{2}-C_{4}-P$; and the public can benefit more as $\beta P-C_{5}-L^{P}>-L^{P}$. Hence, under the stable state $E_{2}(0,0,1)$, no part would like to unilaterally change its current strategy, and then $E_{2}(0,0,1)$ is a Nash equilibrium. For this case, only the public adopts the positive action of "supervision," other than the local government and enterprises. Therefore, the stable state $E_{2}(0,0,1)$ is not an ideal state, under which the epidemic cannot be controlled effectively, and then public health emergencies will be more serious.

If the conditions in Case 3 are all satisfied, which are equivalent to $W^{G}-\alpha C_{3}>C_{1}-C_{2}, R_{1}-C_{3}+\alpha C_{3}+$ $W^{E}>R_{2}-C_{4}-P$, and $H<C_{5}$, then $E_{7}(1,1,0)$ is the ESS. The strategies in $E_{7}(1,1,0)$ are that the local government decides to govern strictly, and the enterprises choose the 
TABLE 3: Eigenvalues of the Jacobian matrix.

\begin{tabular}{lccc}
\hline Equilibrium points & Eigenvalues $\lambda_{1}$ & Eigenvalues $\lambda_{2}$ & Eigenvalues $\lambda_{3}$ \\
\hline$E_{1}(0,0,0)$ & $W^{G}-C_{1}+P+C_{2}$ & $R_{1}-C_{3}-R_{2}+C_{4}$ & $\beta P-C_{5}$ \\
$E_{2}(0,0,1)$ & $-H+\beta P+W^{G}-C_{1}+C_{2}$ & $P+W^{E}+R_{1}-C_{3}-R_{2}+C_{4}$ & $-\beta P+C_{5}$ \\
$E_{3}(0,1,0)$ & $-\alpha C_{3}+W^{G}-C_{1}+C_{2}$ & $\left(R_{2}-R_{1}\right)+\left(C_{3}-C_{4}\right)(>0)$ & $-C_{5}$ \\
$E_{4}(0,1,1)$ & $-H-\alpha C_{3}+W^{G}-C_{1}+C_{2}$ & $-\left(P+W^{E}+R_{1}-C_{3}-R_{2}+C_{4}\right)$ & $C_{5}(>0)$ \\
$E_{5}(1,0,0)$ & $-\left(W^{G}-C_{1}+P+C_{2}\right)$ & $\left(R_{1}+\alpha C_{3}+W^{E}-C_{3}\right)-\left(R_{2}-P-C_{4}\right)(>0)$ & $H-C_{5}$ \\
$E_{6}(1,0,1)$ & $-\left(-H+\beta P+W^{G}-C_{1}+C_{2}\right)$ & $\left(R_{1}+\alpha C_{3}+W^{E}-C_{3}\right)-\left(R_{2}-P-C_{4}\right)(>0)$ & $-\left(H-C_{5}\right)$ \\
$E_{7}(1,1,0)$ & $-\left(-\alpha C_{3}+W^{G}-C_{1}+C_{2}\right)$ & $-\left(\alpha C_{3}+P+W^{E}+R_{1}-C_{3}-R_{2}+C_{4}\right)$ & $H-C_{5}$ \\
$E_{8}(1,1,1)$ & $-\left(-H-\alpha C_{3}+W^{G}-C_{1}+C_{2}\right)$ & $-\left(\alpha C_{3}+P+W^{E}+R_{1}-C_{3}-R_{2}+C_{4}\right)$ & $-\left(H-C_{5}\right)$ \\
\hline
\end{tabular}

TABLE 4: The condition of evolutionary stability of equilibrium points.

\begin{tabular}{lcc}
\hline Equilibrium points & Conditions & Case number \\
\hline$E_{1}(0,0,0)$ & $W^{G}-C_{1}+P+C_{2}<0, R_{1}-C_{3}-R_{2}+C_{4}<0, \beta P-C_{5}<0$ & 1 \\
$E_{2}(0,0,1)$ & $-H+\beta P+W^{G}-C_{1}+C_{2}<0, P+W^{E}+R_{1}-C_{3}-R_{2}+C_{4}<0,-\beta P+C_{5}<0$ & 2 \\
$E_{7}(1,1,0)$ & $\alpha C_{3}-W^{G}+C_{1}-C_{2}<0,-\left(\alpha C_{3}+P+W^{E}+R_{1}-C_{3}-R_{2}+C_{4}\right)<0, H-C_{5}<0$ & 3 \\
$E_{8}(1,1,1)$ & $H+\alpha C_{3}-W^{G}+C_{1}-C_{2}<0,-\left(\alpha C_{3}+P+W^{E}+R_{1}-C_{3}-R_{2}+C_{4}\right)<0,-H+C_{5}<0$ & 4 \\
\hline
\end{tabular}

"strict implementation" strategy, while the public does not supervise. From the conditions in Case 3, we can observe that the reputation $W^{G}$, which is high enough to satisfy the condition of $W^{G}-\alpha C_{3}>C_{1}-C_{2}$, would prompt the local government to govern strictly; the enterprises would strictly implement the regulations to prevent epidemic since they can get higher payoff $\left(R_{1}-C_{3}+\alpha C_{3}+W^{E}\right)$ when "strict governance" strategy is carried out; however, the public would not like to supervise because of the low rewards $H$ and the high cost of supervision $C_{5}$. In this case, the epidemic can be to some extent controlled due to the positive actions from the local government and enterprises, but the incentive mechanism with low reward cannot motivate the public enough.

If the conditions in Case 4 are all satisfied, which are equivalent to $W^{G}-\alpha C_{3}-H-C_{1}>-C_{2}, R_{1}-C_{3}+\alpha C_{3}+$ $W^{E}>R_{2}-C_{4}-P$, and $H>C_{5}$, then $E_{8}(1,1,1)$ is the ESS. The strategies in $E_{8}(1,1,1)$ are that the local government decides to govern strictly, the enterprises choose the "strict implementation" strategy, and the public would like to supervise. From the conditions in Case 4, we can observe that the payoff $\left(=W^{G}-\alpha C_{3}-H-C_{1}\right)$ of the local government from strict governance is higher than that $\left(=-C_{2}\right)$ from nonstrict governance; the enterprises can get more when they choose the "strict implementation" strategy because $R_{1}-C_{3}+\alpha C_{3}+W^{E}>R_{2}-C_{4}-P$; and the public benefits more as $H-C_{5}>0$. Thus, under the stable state $E_{8}(1,1,1)$, no part would like to unilaterally change its current strategy, and then $E_{8}(1,1,1)$ is an ESS. In this case, rational parties will cooperate in fighting against the epidemics and then the ESS $E_{8}(1,1,1)$ is the ideal stable state for the public health emergency, expected by the whole society.

\section{Numerical Simulations}

By using Matlab software, the dynamic evolution of the strategies of the local government, the enterprises, and the public is simulated in this section. Based on the actual situation of the public health emergency, the parameters in our tripartite evolutionary game are set as follows: $C_{1}=$ $15, C_{2}=12, W^{G}=8, H=2, P=4, W^{E}=3, R_{1}=7, C_{3}=$ $4, R \quad{ }_{2}=10, C_{4}=2, \beta P=2, C_{5}=0.5, \alpha C_{3}=1$, $L^{G}=1$, and $L^{P}=1$. It is not hard to see the above values of parameters meet the condition in Case 4 such that $\mathrm{H}+$ $\alpha C_{3}-W^{G}+C_{1}-C_{2}<0,-\left(\alpha C_{3}+P+W^{E}+R_{1}-C_{3}-\right.$ $\left.R_{2}+C_{4}\right)<0,-H+C_{5}<0$. The unique ESS under this situation is thus $E_{8}(1,1,1)$, whose evolution path is demonstrated in Figure 4.

Next, we conduct several numerical experiments to analyze the impacts of parameters on evolutionary results, including the punishment $P$, rewards $H$, subsidy coefficient $\alpha$, and the supervision cost $C_{5}$.

4.1. The Influence of the Punishment on Evolutionary Results. In this subsection, we fix other parameters and let the initial probability $x, y$, and $z$ be 0.5 . The impact of punishment $P$ on the evolutionary results of the enterprises' strategic choice behaviors is shown in Figure 5, in which $P$ is set as 2, 4 , and 6, respectively. From Figure 5, we can observe that with the increase in the punishment, the rate that the enterprises evolve to the "strict implementation" strategy gradually increases. More precisely, when $P$ is increased from 2 to 4 , the length of time for enterprises to reach the stable state of strict implementation is greatly shortened. When $P$ continues to increase from 4 to 6 , although the speed of the enterprise's evolution continues to increase, its strategy adjustment speed is not as rapid as the former. Such an observation illustrates that the local government's punishment measure can promote the strict implementation of enterprises; but its influence is gradually weakened with the punishment increasing.

4.2. The Influence of Rewards on Evolutionary Results. With other parameters being held constant, the impact of rewards $H$ on the evolutionary results of the local government and the public strategy selection is shown in 


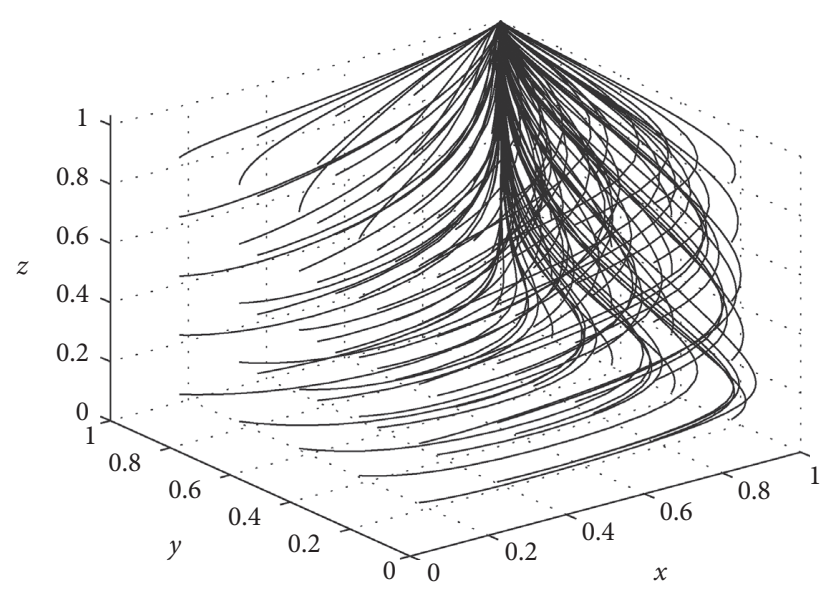

FIgURE 4: Dynamic evolution of three parts' decision-making.

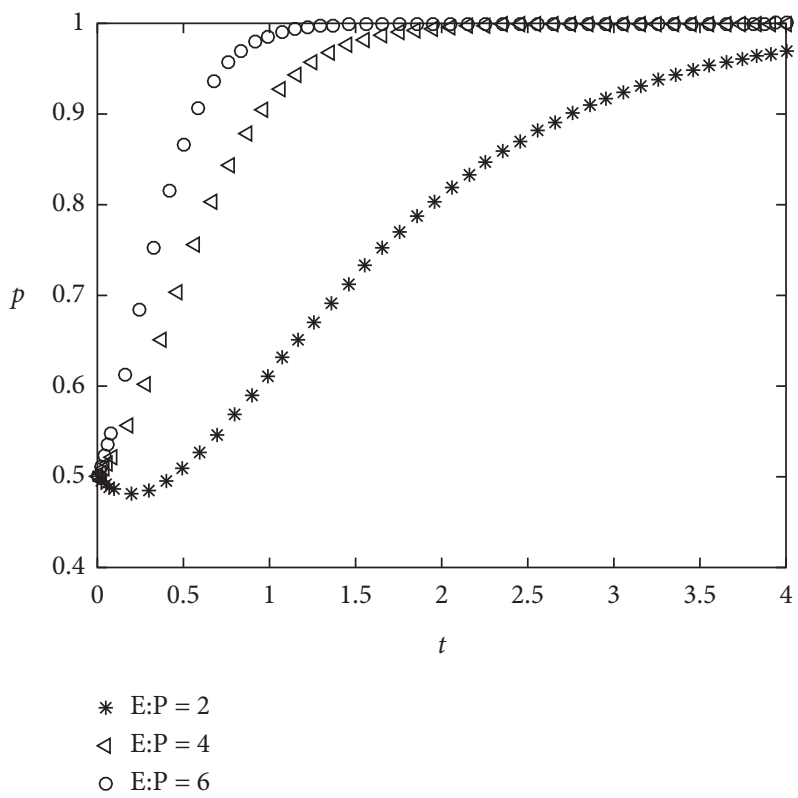

Figure 5: The impact of punishment on the enterprises' decision-making.

Figure 6 . In this experiment, $H$ is set as 1,2 , and 3 , respectively, and the initial probabilities $x, y$, and $z$ are all 0.5 . With the increase in rewards $H$, the public's incentive to supervise also increases. However, the growth rate of the incentive effect on the public gradually weakens, as the rewards continuous to increase (see Figure 6(b)). On the other hand, for the local government, the increase in rewards gradually slows down the evolution speed to the strict governance strategy because high rewards bring heavy pressure on local government's fiscal expenditures. Therefore, a proper reward measure can prompt the public to select the supervision strategy in a short term and enable the government to make proper decision by taking into account the actual fiscal expenditure.

4.3. The Influence of Subsidy Coefficient on Evolutionary Results. To derive the impact of the subsidy coefficient $\alpha$ on evolutionary results, we fix other parameters and conduct experiments when $\alpha=1 / 8,3 / 8$, and 5/8, respectively, and all initial probabilities $x=y=z=0.5$. The dynamic evolutionary processes of the local government and the enterprises are demonstrated in Figure 7. From Figure 7(b), it is easy to see that the subsidy can effectively promote the enterprisers to select the "strict implementation" strategy. Considering to maximize the payoff, the willingness to choose strict implementation strategy would increase with the increase in the subsidy. However, the high subsidy brings a heavy burden on the local government. Thus, the willingness of the government to adopt strict governance would decline with the increase in the subsidy, shown in Figure 7(a). Our experiments indicate that the subsidy measure has an incentive effect on enterprises and draws their attention to the public health emergencies, which prompts them to strictly implement the regulations to prevent and control the epidemic. 


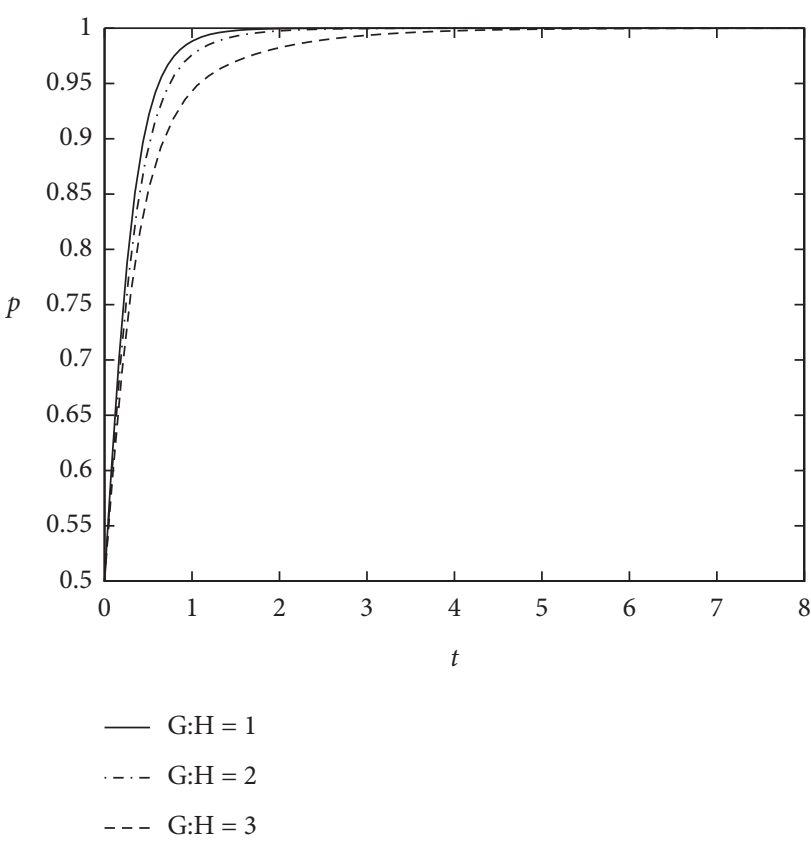

(a)

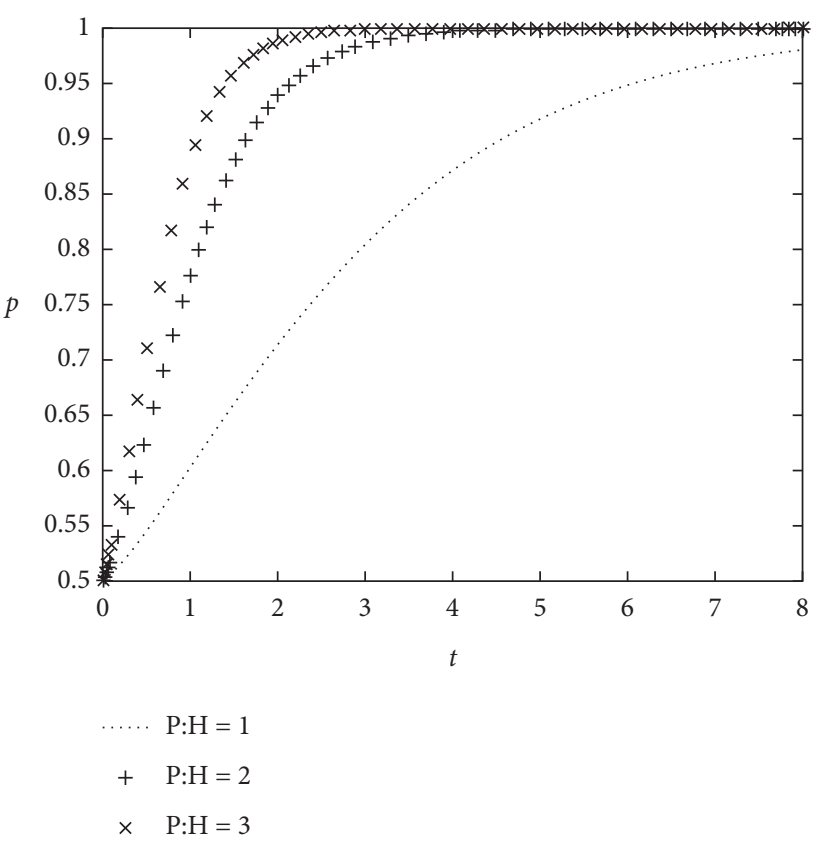

(b)

FIgURE 6: The impact of rewards. Evolutionary behavior of (a) the local government and (b) the public.

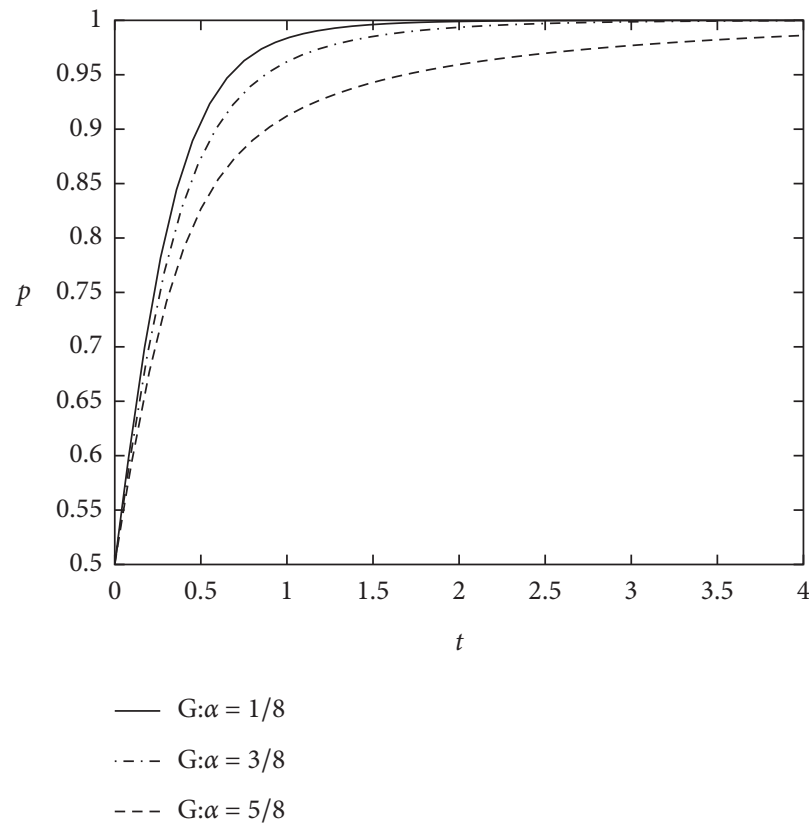

(a)

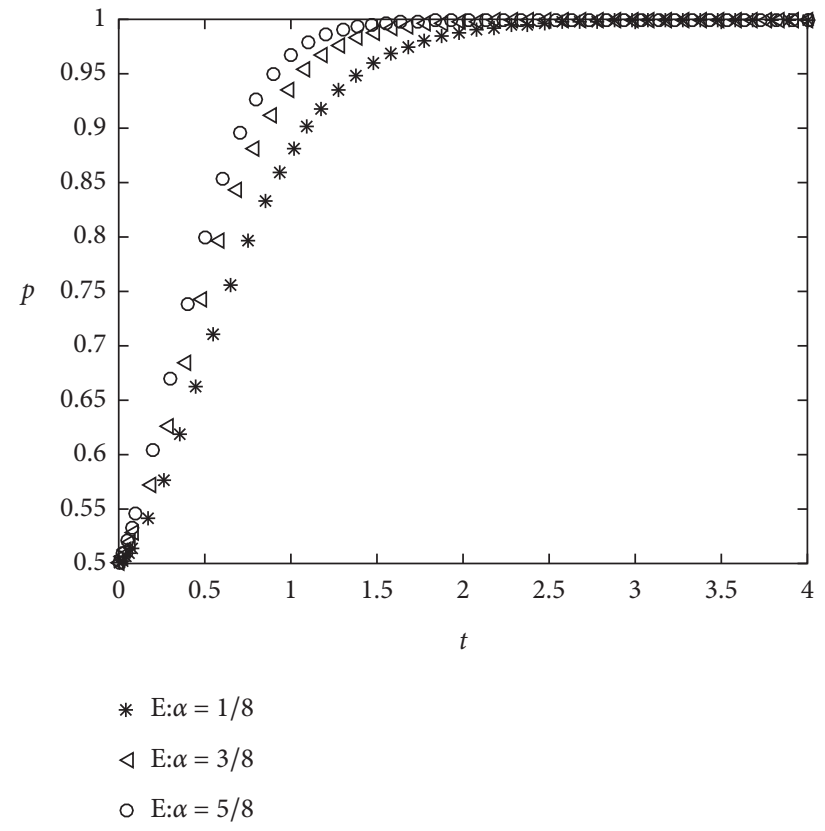

(b)

Figure 7: The impact of subsidy coefficient. Evolutionary behavior of (a) the local government and (b) enterprises.

4.4. The Influence of Supervision Cost on Evolutionary Results. We also fix other parameters, and let the cost $C_{5}$ of supervision be $0.5,1.5$, and 2.5 , respectively, to observe the impact of $C_{5}$ on the strategy selection of the public. Similarly, the initial probabilities $x, y$, and $z$ are set to be 0.5 . It is not hard to find from Figure 8 that the higher the supervision cost is, the less the supervision willingness of the public will be. Particularly, once the cost $C_{5}$ is higher than the reward $H$, for example, $C_{5}=2.5>2=H$, then the public will no longer take the supervision strategy. As the cost of 


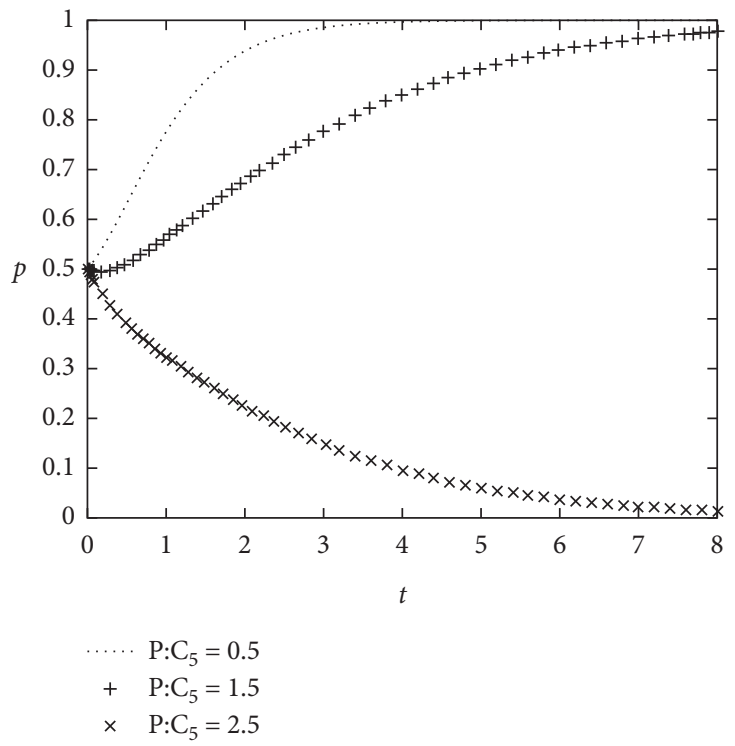

Figure 8: The impact of supervision cost on evolutionary behavior of the public.

supervision decreases, the public will turn to active supervision strategies under the consideration of their benefits and public health.

\section{Conclusion}

Given the actual situation of the public health emergency, a tripartite evolutionary game model is developed for the perspective of cooperation among the local government, the enterprises, and the public. This work studies the factors and evolution path that affect the decision-making of the participants and subsequently verify them by applying numerical simulation technology. Some important conclusions and suggestions are as follows.

As the manager and promoter of epidemic prevention, the government should reinforce the intensity of its supervision and formulate reasonable regulatory measures to deal with public health emergencies timely. It also should avoid overly tough measures, which may cause resistance from the public and enterprises. Training activities on epidemic prevention knowledge for enterprises and the public should be carried out to enhance the awareness of the incident.

The local government should formulate different penalty mechanisms and support mechanisms, while not only the punishment and the subsidy. For example, once the enterprises' negative behaviors are found, the local government shall deal with the offending enterprise immediately. The local government shall pay full attention to the negative impact of the epidemic on enterprises' production and operation. While maintaining social security and stability, the government shall protect enterprises' interests, care about their production and operation conditions, and know about their actual conditions such as capital operation, rent, production, and sales. Promoting coordination and cooperation between the government and enterprises can ultimately achieve mutual benefit and win-win results.
In addition, the government shall try the best to reduce the public's supervision cost and facilitate the supervision of the public through multiple channels to improve the public's participation in public health emergencies.

\section{Data Availability}

The data used to support the findings of this study are included within the article.

\section{Conflicts of Interest}

The authors declare that they have no conflicts of interest.

\section{Acknowledgments}

This research was supported by the National Nature Science Foundation of China (nos. 11871366 and 61803279) and Qing Lan Project for Young Academic Leaders, Qing Lan Project for Key Teachers, and the Research Innovation Program for College Graduate Students of Jiangsu Province (no. KYCX20-2790).

\section{References}

[1] https://coronavirus.jhu.edu/map.html.

[2] https://www.who.int/news-room/detail/30-01-2020statement-on-the-second-meeting-of-the-internationalhealth-regulations-(2005)-emergency-committee-regardingthe-outbreak-of-novel-coronavirus-(2019-ncov).

[3] https://www.aa.com.tr/en/health/no-vaccine-specific-drugagainst-coronavirus-yet-who/ 1803428 .

[4] W. O. Kermack and A. G. McKendrick, "A contribution to the mathematical theory of epidemics," Proceedings of the Royal Society of London. Series A, Containing Papers of a Mathematical and Physical Character, vol. 115, no. 772, pp. 700-721, 1927. 
[5] J. Saramäki and K. Kaski, "Modelling development of epidemics with dynamic small-world networks," Journal of Theoretical Biology, vol. 234, no. 3, pp. 413-421, 2005.

[6] X.-J. Xu, H.-O. Peng, X.-M. Wang, and Y.-H. Wang, "Epidemic spreading with time delay in complex networks," Physica A: Statistical Mechanics and Its Applications, vol. 367, pp. 525-530, 2006.

[7] C. Sun and Y.-H. Hsieh, "Global analysis of an SEIR model with varying population size and vaccination," Applied Mathematical Modelling, vol. 34, no. 10, pp. 2685-2697, 2010.

[8] J. Zhang, J. Li, and Z. Ma, "Global dynamics of an SEIR epidemic model with immigration of different compartments," Acta Mathematica Scientia, vol. 26, no. 3, pp. 551-567, 2006.

[9] C. Hou, J. Chen, Y. Zhou et al., "The effectiveness of quarantine of Wuhan city against the Corona Virus Disease 2019 (COVID 19): a well-mixed SEIR model analysis," Journal of Medical Virology, vol. 92, no. 7, 2020.

[10] K. Prem, Y. Liu, T. W. Russell et al., "The effect of control strategies to reduce social mixing on outcomes of the COVID19 epidemic in Wuhan, China: a modelling study," The Lancet Public Health, vol. 5, 2020.

[11] A. Radulescu and K. Cavanagh, "Management strategies in a SEIR model of COVID 19 community spread," 2020, https:// arxiv.org/abs/2003.11150.

[12] J. Zu, M. L. Li, Z. F. Li et al., "Transmission patterns of COVID-19 in the mainland of China and the efficacy of different control strategies: a data-and model-driven study," Infectious Diseases of Poverty, vol. 9, no. 1, pp. 1-14, 2020.

[13] C. Li, F. Zhang, C. Cao, Y. Liu, and T. Qu, "Organizational coordination in sustainable humanitarian supply chain: an evolutionary game approach," Journal of Cleaner Production, vol. 219, pp. 291-303, 2019.

[14] Z. Wang, W. Xu, J. Yang et al., "A game theoretic approach for resource allocation based on ant colony optimization in emergency management," in Proceedings of the 2009 International Conference on Information Engineering and Computer Science, pp. 1-4, Wuhan, China, October 2009.

[15] J.-J. Yang and C.-H. Xu, "Emergency decision engineering model based on sequential games," Systems Engineering Procedia, vol. 5, pp. 276-282, 2012.

[16] S. Purohit and S. Mantri, "Game theoretic multi-agent approach to traffic flow control," in Proceedings of the 2013 International Conference on Advances in Computing, Communications and Informatics (ICACCI), pp. 1902-1905, Mysore, India, August 2013.

[17] L. Hongyan, "Evolutionary game analysis of emergency management of the Middle Route of South-to-North Water diversion Project," Water Resources Management, vol. 31, no. 9, pp. 2777-2789, 2017.

[18] https://www.chinadaily.com.cn/a/202012/04/ WS5fca4e25a31024ad0ba99e75.html.

[19] P. D. Taylor and L. B. Jonker, "Evolutionary stable strategies and game dynamics," Mathematical Biosciences, vol. 40, no. 12, pp. 145-156, 1978.

[20] S. Y. Xie, The Game of Economices, Fudan University Press, Shanghai, China, 2010.

[21] R. Selten, "A note on evolutionarily stable strategies in asymmetric animal conflicts," 1978.

[22] K. Ritzberger and J. W. Weibull, "Evolutionary selection in normal-form games," Journal of the Econometric Society, vol. 63, no. 6, pp. 1371-1399, 1995.

[23] H. K. Khalil and J. W. Grizzle, Nonlinear Systems, PrenticeHall, ” Upper Saddle River, NJ, USA, 2002. 